\title{
Construction of generalized coordinates' basis functions in Lagrangian dynamics of flat manipulators
}

\author{
Bagautdinov Ildar Nyrgaiazovich ${ }^{1, *}$, Pavlov Alexander Ivanovich ${ }^{2}$, \\ Zhuravlev Evgeny Alekseevich $^{1}$, Bogdanov Evgeny Nikolaevich ${ }^{1}$ \\ ${ }^{1}$ Faculty of Mechanics and Machine Building of Volga State University of Technology, Yoshkar-Ola, Russia \\ ${ }^{2}$ Professor of Syktyvkar State University, Syktyvkar, Russia
}

\section{Email address:}

BagautdinovIN@volgatech.net (Bagautdinov I.N.)

\section{To cite this article:}

Bagautdinov Ildar Nyrgaiazovich, Pavlov Alexander Ivanovich, Zhuravlev Evgeny Alekseevich, Bogdanov Evgeny Nikolaevich. Construction of Generalized Coordinates' Basis Functions in Lagrangian Dynamics of Flat Manipulators. Applied and Computational Mathematics. Vol. 3, No. 4, 2014, pp. 186-190. doi: 10.11648/j.acm.20140304.20

\begin{abstract}
Second order Lagrange equations are used for describing dynamics of planar mechanism with rotation joints. For calculating kinetic energy of the links local coordinates of velocity vectors are used as well as recursive matrix transformations. Kinetic energy quadratic form coefficients are represented by linear combinations of seven independent trigonometric functions of generalized coordinates, i.e. basis functions. A number of these functions are connected to number of links by quadratic dependence. Constant coefficients in expansions in basic functions are determined from linear equation systems, representing kinetic energy of the mechanism in its several nonrecurring configurations with non-zero values for one or two generalized velocities. The resulting system of dynamics differential equations is integrated numerically with Runge-Kutta method in software environment Mathcad. Efficiency of the proposed method of creating and solving dynamic equations is demonstrated by example of numerical solution the direct dynamic problem of three-link mechanism.
\end{abstract}

Keywords: Flat Multilink Mechanism, Lagrange Equations, Basis Functions, Direct Dynamic Problem

\section{Introduction}

Voluminous literature is devoted to methods of creating equations of the joined multilink mechanisms dynamics, which are the basis of industrial robot manipulating systems. One of the main objectives for the authors, focused on this problem, is to create a most effective algorithm for building-up dynamic equations for those kinds of mechanisms. Contrastive analysis [1] shows a significant dependence of different approaches efficiency on the $\mathrm{N}$ number of links in kinematic chain of the mechanism and its geometry; for $\mathrm{N}=2-6$ Lagrange description of mechanism dynamics is entirely acceptable.

In this paper differential dynamics equations of manipulator are built-up on the basis of second order Lagrange equations. It is commonly known that kinetic energy of rigid-body mechanism with finite number of degrees of freedom represents positively definite quadratic form of generalized velocities. Its coefficients are regarded as functions of mechanism generalized coordinates and serve as components of inertia matrix of manipulator. This paper shows that all components of inertia matrix of a planar manipulator with rotating pairs can be represented as linear combinations of some set of linearly independent trigonometric functions of generalized coordinates. By analogy with some properties of vector spaces, these functions are defined here [3] as basic functions.

This paper adduces detailed arguments in favor of basic functions' existence and gives the algorithm of their construction for planar n-link manipulator. Alongside, we provide the algorithm of finding numerical coefficients at basic functions in linear combinations representing inertia matrix elements. Essentially, these coefficients are constant and invariant with mechanism configuration, thus, they are calculated only once during constructing dynamic model of the manipulator.

Introduction of inertia matrix elements as linear combinations of basic functions makes algorithm of forming the system of differential equations of mechanism dynamics much easier. 


\section{Building Mathematical Model}

We consider the motion of a planar multilink mechanism, pivoted with fixed base (figure 1). All links are absolutely solid objects, changing their position in horizontal plane under the action of moments $M_{1}, M_{2}, \ldots M_{n}$ in knuckle joints $O_{1}, O_{2}, O_{n}$ (Figure 1). Connect every link with local system of coordinates $O_{k} x_{k} y_{k}$, where axis $O_{k} x_{k}$ passes through axes of component joints, and for the final link $(k=$ 3 ) it is pointed arbitrary (Figure 1); $O_{0} x_{0} y_{0}$ - is a fixed inertial coordinate system. Identified: components' mass $m_{k}$, position of their mass centers $C_{k}$ and central moments of inertia $I_{k}$ about the axes, vertical to the motion plane; consider these axes as links inertia main axes.

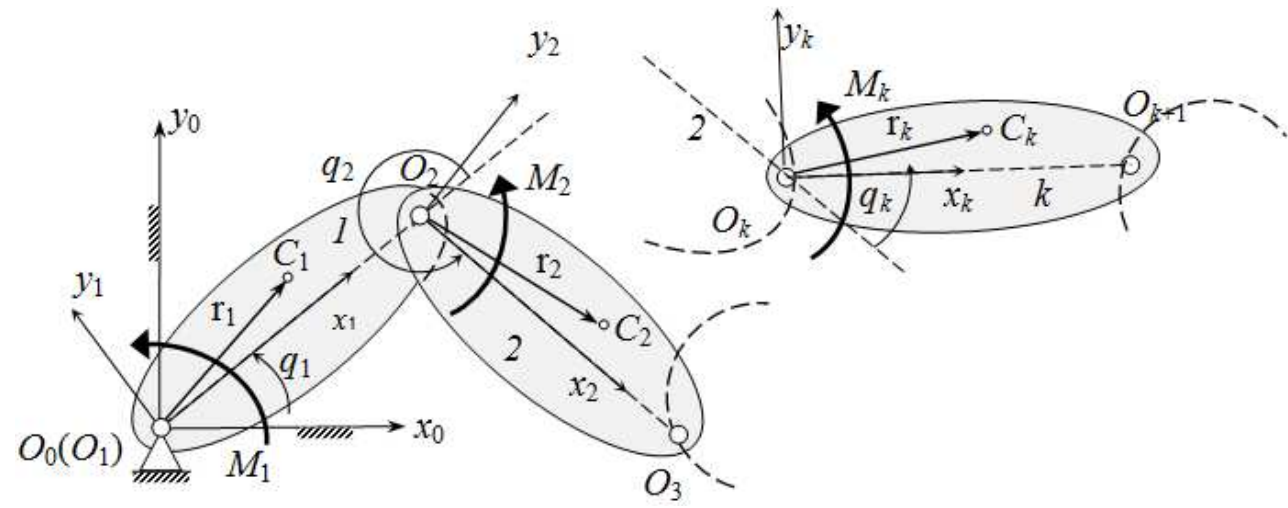

Figure 1. Computational scheme of mechanism

The angles of reciprocal link rotation $\mathrm{q} 1, \mathrm{q} 2, \mathrm{q} 3$, measured counterclockwise, are taken as generalized coordinates (figure 1)

Kinetic energy $\mathrm{T}$ of the mechanism is composed of kinetic energy of its links:

$$
T=\frac{1}{2} \sum_{k=1}^{n}\left(m_{k} \mathbf{v}_{k}^{2}+I_{k} \omega_{k}^{2}\right)
$$

where vk - mass center velocity of a k-link, and

$$
\omega_{k}=\sum_{m=1}^{k} \dot{q}_{m}
$$

angle speed of $k$ link in fixed frame of reference $O_{0} x_{0} y_{0}$.

Kinetic energy of the considered mechanical system with finite number of degrees of freedom is positively definite quadratic form of generalized velocities. Coefficients of this form depend only on generalized coordinates. [4]

$$
\mathrm{T}=\frac{1}{2} \sum_{\mathrm{i}=1}^{\mathrm{n}} \sum_{\mathrm{j}=1}^{\mathrm{n}} \mathrm{a}_{\mathrm{ij}}(\mathrm{q}) \dot{\mathrm{q}}_{\mathrm{i}} \dot{\mathrm{q}}_{\mathrm{j}}, \quad \mathrm{q}=\left(\mathrm{q}_{1}, \mathrm{q}_{2}, \ldots, \mathrm{q}_{\mathrm{n}}\right) .
$$

In order to study the structure of expressions $a_{i j}(\mathrm{q})$ assume velocities of links mass centers $\mathrm{v}_{k}$ as recursive relations.

$$
\begin{gathered}
\mathrm{v}_{\mathrm{k}}=\mathrm{u}_{\mathrm{k}-1}+\omega_{\mathrm{k}} \times \mathrm{r}_{\mathrm{k}},(\mathrm{k}=1,2, \ldots, \mathrm{n}) ; \\
\mathrm{u}_{0}=0, \mathrm{u}_{\mathrm{m}}=\mathrm{u}_{\mathrm{m}-1}+\omega_{\mathrm{m}} \times \mathrm{L}_{\mathrm{m}}, \\
(\mathrm{m}=1,2, \ldots, \mathrm{n}-1),
\end{gathered}
$$

where $\mathrm{r}_{k}=\overline{O_{k} C_{k}}$ - radius-vectors of links' mass centers; $L_{m}=\overline{O_{m} O_{m+1}}$ - radius-vectors of knuckle joints' centers of links; $\mathrm{u}_{m}-$ speeds of joints' centers $O_{m} ; \omega_{k}-$ angular velocity vector of the $k$ link.

While using flat coordinate system $O_{k} x_{k} y_{k}$ and twocomponent vectors it is easy to demonstrate (4) in matrix form

$$
\begin{gathered}
\mathrm{v}_{k}=\mathrm{T}_{k} \mathrm{u}_{k-1}+\Omega_{k} \mathrm{r}_{k}, \quad(\mathrm{k}=1,2, \ldots, n) ; \\
\mathrm{u}_{0}=0, \mathrm{u}_{\mathrm{m}}=\mathrm{T}_{\mathrm{m}} \mathrm{u}_{\mathrm{m}-1}+\Omega_{\mathrm{m}} \times \mathrm{L}_{\mathrm{m}},(\mathrm{m}=1,2, \ldots, \mathrm{n}-1)
\end{gathered}
$$

Here, coordinates of speed vectors $\mathrm{v}_{k}$ and $\mathrm{u}_{m}$ are shown in local coordinate systems $O_{k} x_{k} y_{k}$ and $O_{m} x_{m} y_{m}$ respectively; $\mathrm{T}_{k}=\left(\begin{array}{cc}\cos q_{k} & \sin q_{k} \\ -\sin q_{k} & \cos q_{k}\end{array}\right)$ - vector coordinate transformation matrix out of the system $O_{k-1} x_{k-1} y_{k-1}$ в $O_{k} x_{k} y_{k} ; \Omega_{k}=\left(\begin{array}{cc}0 & -\omega_{k} \\ \omega_{k} & 0\end{array}\right)$ - skew symmetric matrix of the angular velocity for $k$ link; $\mathrm{L}_{m}=\left(L_{m}, 0\right)^{\mathrm{T}} ; \mathrm{r}_{k}=\left(r_{k x}, r_{k y}\right)^{\mathrm{T}}$.

Consequent application of (5) gives matrix expression for center mass velocities of links:

$$
\begin{gathered}
\mathrm{v}_{1}=\Omega_{1} \mathrm{r}_{1}, \\
\mathrm{v}_{2}=\mathrm{T}_{2} \Omega_{1} \mathrm{~L}_{1}+\Omega_{2} \mathrm{r}_{2}, \mathrm{v}_{3}=\mathrm{T}_{3}\left(\mathrm{~T}_{2} \Omega_{1} \mathrm{~L}_{1}+\Omega_{2} \mathrm{~L}_{2}\right)+\Omega_{3} \mathrm{r}_{3}, \ldots \\
\mathrm{v}_{\mathrm{n}}=\mathrm{T}_{\mathrm{n}} \mathrm{T}_{\mathrm{n}-1} \cdots \mathrm{T}_{2} \Omega_{1} \mathrm{~L}_{1}+\mathrm{T}_{\mathrm{n}} \mathrm{T}_{\mathrm{n}-1} \cdots \mathrm{T}_{3} \Omega_{2} \mathrm{~L}_{2}+ \\
\cdots+\mathrm{T}_{\mathrm{n}} \mathrm{T}_{\mathrm{n}-1} \Omega_{\mathrm{n}-2} \mathrm{~L}_{\mathrm{n}-2}+\mathrm{T}_{\mathrm{n}} \Omega_{\mathrm{n}-1} \mathrm{~L}_{\mathrm{n}-1}+\Omega_{\mathrm{n}} \mathrm{r}_{\mathrm{n}}
\end{gathered}
$$

or in component-wise message

$$
\mathrm{v}_{1}=\omega_{1}\left(\begin{array}{c}
-r_{1 y} \\
r_{1 x}
\end{array}\right)
$$

$$
v_{2}=\omega_{1} L_{1}\left(\begin{array}{c}
\sin q_{2} \\
\cos q_{2}
\end{array}\right)+\omega_{2}\left(\begin{array}{c}
-r_{2 y} \\
r_{2 x}
\end{array}\right) \text {, }
$$




$$
\begin{gathered}
\mathrm{v}_{3}=\omega_{1} \mathrm{~L}_{1}\left(\begin{array}{c}
\sin \left(\mathrm{q}_{2}+\mathrm{q}_{3}\right) \\
\cos \left(\mathrm{q}_{2}+\mathrm{q}_{3}\right)
\end{array}\right)+\omega_{2} \mathrm{~L}_{2}\left(\begin{array}{c}
\sin \mathrm{q}_{3} \\
\cos \mathrm{q}_{3}
\end{array}\right)+\omega_{3}\left(\begin{array}{c}
-\mathrm{r}_{3 \mathrm{y}} \\
\mathrm{r}_{3 \mathrm{x}}
\end{array}\right), \ldots \\
\mathrm{v}_{\mathrm{n}}=\omega_{1} \mathrm{~L}_{1}\left(\begin{array}{c}
\sin \left(\mathrm{q}_{2}+\cdots+\mathrm{q}_{\mathrm{n}}\right) \\
\cos \left(\mathrm{q}_{2}+\cdots+\mathrm{q}_{\mathrm{n}}\right)
\end{array}\right)+\omega_{2} \mathrm{~L}_{2}\left(\begin{array}{c}
\sin \left(\mathrm{q}_{3}+\cdots+\mathrm{q}_{\mathrm{n}}\right) \\
\cos \left(\mathrm{q}_{3}+\cdots+\mathrm{q}_{\mathrm{n}}\right)
\end{array}\right)+ \\
\cdots+\omega_{\mathrm{n}-1} \mathrm{~L}_{\mathrm{n}-1}\left(\begin{array}{c}
\sin \mathrm{q}_{\mathrm{n}} \\
\cos \mathrm{q}_{\mathrm{n}}
\end{array}\right)+\omega_{\mathrm{n}}\left(\begin{array}{c}
-\mathrm{r}_{\mathrm{ny}} \\
\mathrm{r}_{\mathrm{n}}
\end{array}\right)
\end{gathered}
$$

Using (6), certain rules of matrix algebra and trigonometric identities we get the following expressions for squared velocities of links' mass centers:

$$
\begin{aligned}
& v_{1}^{2}=\omega_{1}^{2} r_{1}^{2} \\
& v_{2}^{2}=\omega_{2}^{2} r_{2}^{2}+\omega_{1}^{2} L_{1}^{2}+2 \omega_{1} \omega_{2} L_{1}\left(r_{2 x} \cos q_{2}-r_{2 y} \sin q_{2}\right) \\
& \mathrm{v}_{3}^{2}=\omega_{1}^{2} \mathrm{~L}_{1}^{2}+\omega_{2}^{2} \mathrm{~L}_{2}^{2}+\omega_{3}^{2} \mathrm{r}_{3}^{2}+2 \omega_{3}\left\{\omega _ { 1 } \mathrm { L } _ { 1 } \left[\mathrm{r}_{\mathrm{nx}} \cos \left(\mathrm{q}_{2}+\mathrm{q}_{3}\right)\right.\right. \\
& \left.-\mathrm{r}_{\mathrm{ny}} \sin \left(\mathrm{q}_{2}+\mathrm{q}_{3}\right)\right]+ \\
& \left.+\omega_{2} \mathrm{~L}_{2}\left[\mathrm{r}_{\mathrm{nx}} \cos \mathrm{q}_{3}-\mathrm{r}_{\mathrm{ny}} \sin \mathrm{q}_{3}\right]\right\}+2 \omega_{1} \mathrm{~L}_{1} \omega_{2} \mathrm{~L}_{2} \cos \mathrm{q}_{2}, \ldots \\
& \mathrm{v}_{\mathrm{k}}^{2}=\omega_{1}^{2} \mathrm{~L}_{1}^{2}+\cdots+\omega_{\mathrm{k}-1}^{2} \mathrm{~L}_{\mathrm{k}-1}^{2}+\omega_{\mathrm{k}}^{2} \mathrm{r}_{\mathrm{k}}^{2}+2 \omega_{\mathrm{k}}\left\{\omega_{1} \mathrm{~L}_{1}[\right. \\
& \left.\mathrm{r}_{\mathrm{kx}} \cos \left(\mathrm{q}_{2}+\cdots+\mathrm{q}_{\mathrm{k}}\right)-\mathrm{r}_{\mathrm{ky}} \sin \left(\mathrm{q}_{2}+\cdots+\mathrm{q}_{\mathrm{k}}\right)\right]+ \\
& +\omega_{2} \mathrm{~L}_{2}\left[\mathrm{r}_{\mathrm{kx}} \cos \left(\mathrm{q}_{3}+\cdots+\mathrm{q}_{\mathrm{k}}\right)-\mathrm{r}_{\mathrm{ky}} \sin \left(\mathrm{q}_{3}+\cdots+\mathrm{q}_{\mathrm{k}}\right)\right]+ \\
& \left.\cdots+\omega_{\mathrm{k}-1} \mathrm{~L}_{\mathrm{k}-1}\left(\mathrm{r}_{\mathrm{kx}} \cos \mathrm{q}_{\mathrm{k}}-\mathrm{r}_{\mathrm{ky}} \sin \mathrm{q}_{\mathrm{k}}\right)\right\}+ \\
& +2 \omega_{1} \mathrm{~L}_{1}\left[\omega_{2} \mathrm{~L}_{2} \cos \mathrm{q}_{2}+\omega_{3} \mathrm{~L}_{3} \cos \left(\mathrm{q}_{2}+\mathrm{q}_{3}\right)+\right. \\
& \left.\cdots+\omega_{\mathrm{n}-1} \mathrm{~L}_{\mathrm{n}-1} \cos \left(\mathrm{q}_{2}+\cdots+\mathrm{q}_{\mathrm{k}-1}\right)\right]+ \\
& +2 \omega_{2} \mathrm{~L}_{2}\left[\omega_{3} \mathrm{~L}_{3} \cos \mathrm{q}_{3}+\omega_{4} \mathrm{~L}_{4} \cos \left(\mathrm{q}_{3}+\mathrm{q}_{4}\right)+\right. \\
& \left.\cdots+\omega_{\mathrm{n}-1} \mathrm{~L}_{\mathrm{n}-1} \cos \left(\mathrm{q}_{3}+\cdots+\mathrm{q}_{\mathrm{k}-1}\right)\right]+\cdots \\
& +2 \omega_{\mathrm{k}-3} \mathrm{~L}_{\mathrm{k}-3}\left[\omega_{\mathrm{k}-2} \mathrm{~L}_{\mathrm{k}-2} \cos \mathrm{q}_{\mathrm{k}-2}+\right. \\
& \left.\omega_{\mathrm{k}-1} \mathrm{~L}_{\mathrm{k}-1} \cos \left(\mathrm{q}_{\mathrm{k}-2}+\mathrm{q}_{\mathrm{k}-1}\right)\right]+2 \omega_{\mathrm{k}-2} \mathrm{~L}_{\mathrm{k}-2} \omega_{\mathrm{k}-1} \mathrm{~L}_{\mathrm{k}-1} \cos \mathrm{q}_{\mathrm{k}-1}
\end{aligned}
$$

Equality (7) detects that expression for kinetic energy of a separated $k$-link of the manipulator, coefficients at generalized velocities $\mathrm{q}_{1}, \ldots, \mathrm{q}_{\mathrm{k}}$ include linear combinations only of the following functions of generalized coordinates:

$$
\begin{aligned}
& 1, \sin \left(\mathrm{q}_{2}+\mathrm{q}_{3}+\cdots+\mathrm{q}_{\mathrm{k}}\right), \sin \left(\mathrm{q}_{3}+\cdots+\mathrm{q}_{\mathrm{k}}\right), \ldots, \sin \mathrm{q}_{\mathrm{k}}, \\
& \cos \left(\mathrm{q}_{2}+\mathrm{q}_{3}+\cdots+\mathrm{q}_{\mathrm{k}}\right), \cos \left(\mathrm{q}_{3}+\cdots+\mathrm{q}_{\mathrm{k}}\right), \ldots, \cos \mathrm{q}_{\mathrm{k}}, \\
& \left\{\cos \mathrm{q}_{2}, \cos \left(\mathrm{q}_{2}+\mathrm{q}_{3}\right), \cos \left(\mathrm{q}_{2}+\mathrm{q}_{3}+\cdots+\mathrm{q}_{\mathrm{k}-1}\right),\right. \\
& \cos \mathrm{q}_{3}, \cos \left(\mathrm{q}_{3}+\mathrm{q}_{4}\right), \cos \left(\mathrm{q}_{3}+\mathrm{q}_{4}+\cdots+\mathrm{q}_{\mathrm{k}-1}\right), \\
& \ldots \ldots \cdots \cdots, \\
& \left.\cos \mathrm{q}_{\mathrm{k}-2}, \cos \left(\mathrm{q}_{\mathrm{k}-2}+\mathrm{q}_{\mathrm{k}-1}\right), \cos \mathrm{q}_{\mathrm{k}-1}\right\} .
\end{aligned}
$$

It is easy to notice that all functions in (8), enclosed in braces, appear in kinetic energy expression of previous links $k-1, k-2, \quad, 3,2$.

In terms of the conducted analysis, we are able to consolidate that quadric form coefficients, expressing kinematic energy of the whole n-link manipulator, represent linear combinations of the following linearly independent functions

$1, \cos q_{2}, \sin q_{2}$,

$\cos \mathrm{q}_{3}, \sin \mathrm{q}_{3}, \cos \left(\mathrm{q}_{2}+\mathrm{q}_{3}\right), \sin \left(\mathrm{q}_{2}+\mathrm{q}_{3}\right)$,

$\cos q_{4}, \sin \mathrm{q}_{4}, \cos \left(\mathrm{q}_{3}+\mathrm{q}_{4}\right), \sin \left(\mathrm{q}_{3}+\mathrm{q}_{4}\right)$, $\cos \left(\mathrm{q}_{2}+\mathrm{q}_{3}+\mathrm{q}_{4}\right), \sin \left(\mathrm{q}_{2}+\mathrm{q}_{3}+\mathrm{q}_{4}\right)$,

$\cdots \ldots$,

$\cos \mathrm{q}_{\mathrm{n}}, \sin \mathrm{q}_{\mathrm{n}}, \cos \left(\mathrm{q}_{\mathrm{n}-1}+\mathrm{q}_{\mathrm{n}}\right), \sin \left(\mathrm{q}_{\mathrm{n}-1}+\mathrm{q}_{\mathrm{n}}\right), \ldots$, $\cos \left(\mathrm{q}_{2}+\cdots+\mathrm{q}_{\mathrm{n}}\right), \sin \left(\mathrm{q}_{2}+\cdots+\mathrm{q}_{\mathrm{n}}\right)$,

They will be called basic functions and take the symbols of $\alpha_{1}, \alpha_{2}, \ldots, \alpha_{m}$. Number $m$ of the basic functions is connected to $n$ - the number of links of manipulator by the formula $\mathrm{m}=1+\mathrm{n}(\mathrm{n}-1)$.

Coefficients $a_{i j}(\mathrm{q})$ of the quadric form (3), which are proved to be the components of the inertia matrix of the manipulator can be assumed in the following equation:

$$
\mathrm{a}_{\mathrm{ij}}(\mathrm{q})=\mathrm{a}_{\mathrm{ij}}\left(\mathrm{q}_{2}, \ldots, \mathrm{q}_{\mathrm{n}}\right)=\sum_{\mathrm{s}=1}^{\mathrm{m}} \mathrm{c}_{\mathrm{s}}^{(\mathrm{ij})} \alpha_{\mathrm{s}}\left(\mathrm{q}_{2}, \ldots, \mathrm{q}_{\mathrm{n}}\right),(i, j=1,2, \ldots,
$$

In order to find constants $c_{s}^{(i j)}$ in (9), we take advantage of the mode, recommended in the paper [5] for calculating elements of the inertia matrix of joint objects.

Denote $T_{i j}(q)$ as a kinetic energy rate of mechanical system, positioned in random preset configuration $\mathrm{q}=\left(\mathrm{q}_{1}, \mathrm{q}_{2}, \ldots, \mathrm{q}_{\mathrm{n}}\right)$ that has generalized velocities $\dot{q}_{i}=\dot{q}_{j}=1, \dot{q}_{k}=0,(k \neq i, j)$. Values $\mathrm{T}_{\mathrm{ij}}$ (q) are quite easy to compute with the help of relations obtained previously (1), (2) и (6). Subsequently, numeric values of inertia matrix elements $a_{i j}(q)$ for this configuration can be logically found with the following equations:

$$
\begin{gathered}
a_{i i}(q)=2 T_{i i}(q), \quad a_{i j}(q)=2 T_{j j}(q), \\
a_{i j}(q)=T_{i j}(q)-T_{i i}(q)-T_{j j}(q), \quad(i, j=1,2, \ldots, n),
\end{gathered}
$$

resulted from (3).

Suppose, values $a_{i j}(q)$ are determined for $m$ different configurations $\mathrm{q}_{1}, \mathrm{q}_{2}, \ldots, \mathrm{q}_{\mathrm{m}}$ of the mechanical system under the analysis, then the constants $c_{S}^{(i j)}(s=1, \ldots, m)$ in expansion (9) can be found by solving the system $m$ of linear algebraic equations

$$
\sum_{\mathrm{s}=1}^{\mathrm{m}} \alpha_{\mathrm{s}}\left(\mathrm{q}_{1}\right) \mathrm{c}_{\mathrm{s}}^{(\mathrm{ij})}=\mathrm{a}_{\mathrm{ij}}\left(\mathrm{q}_{1}\right),(1=1, \ldots, \mathrm{m})
$$


with nonzero determinant $\left|\begin{array}{ccc}\alpha_{1}\left(\mathrm{q}_{1}\right) & \ldots & \alpha_{\mathrm{m}}\left(\mathrm{q}_{1}\right) \\ \ldots & \ldots & \ldots \\ \alpha_{1}\left(\mathrm{q}_{\mathrm{m}}\right) & \ldots & \alpha_{\mathrm{m}}(\mathrm{qm})\end{array}\right|$, where $\mathrm{q}_{1}, \ldots, \mathrm{q}_{\mathrm{m}}$ - various configurations of manipulator. In order to find all $c_{S}^{(i j)}$, taking account of coefficients symmetry $\mathrm{a}_{\mathrm{ij}}(\mathrm{q})$, it is sufficient to form and solve $n(n-1) / 2$ systems of that kind.

For describing dynamics of the mechanism we apply Lagrange equation of the second race:

$$
\frac{\mathrm{d}}{\mathrm{dt}}\left(\frac{\partial \mathrm{T}}{\partial \dot{\mathrm{q}}_{\mathrm{i}}}\right)-\frac{\partial \mathrm{T}}{\partial \mathrm{q}_{\mathrm{i}}}=\mathrm{Q}_{\mathrm{i}},(\mathrm{i}=1,2, \ldots, n),
$$

where $Q_{i}=M_{i}-$ generalized forces, equal to moments, functioning in joints.

Compute derivatives in the left parts of equations (10):

$\frac{d}{d t}\left(\frac{\partial T}{\partial \dot{q}_{i}}\right)-\frac{\partial T}{\partial q_{i}}=\sum_{j=1}^{n} a_{i j} \ddot{q}_{j}+\sum_{j=1}^{n}\left(\sum_{k=1}^{n} \frac{\partial a_{i j}}{\partial q_{k}} \dot{q}_{k}\right) \dot{q}_{j}-\frac{1}{2} \sum_{j=1}^{n} \sum_{k=1}^{n} \frac{\partial a_{j k}}{\partial q_{i}} \dot{q}_{j} \dot{q}_{k}$

We transform it applying (9) and matrix notations:

$$
\begin{aligned}
& \frac{\mathrm{d}}{\mathrm{dt}}\left(\frac{\partial \mathrm{T}}{\partial \dot{\mathrm{q}}_{\mathrm{i}}}\right)-\frac{\partial \mathrm{T}}{\partial \mathrm{q}_{\mathrm{i}}}=\sum_{\mathrm{j}=1}^{\mathrm{n}}\left(\mathrm{a}_{0}^{\mathrm{T}} \mathrm{c}_{\mathrm{ij}}\right) \ddot{\mathrm{q}}_{\mathrm{j}}+\left(\sum_{\mathrm{k}=1}^{\mathrm{n}} \mathrm{a}_{\mathrm{k}} \dot{\mathrm{q}}_{\mathrm{k}}\right)^{\mathrm{T}} \sum_{\mathrm{j}=1}^{\mathrm{n}} \mathrm{c}_{\mathrm{ij}} \dot{\mathrm{q}}_{\mathrm{j}} \\
& -\frac{1}{2} \mathrm{a}_{\mathrm{i}}^{\mathrm{T}} \sum_{\mathrm{j}=1}^{3} \sum_{\mathrm{k}=1}^{3} \mathrm{c}_{\mathrm{jk}} \dot{\mathrm{q}}_{\mathrm{j}} \dot{\mathrm{q}}_{\mathrm{k}}
\end{aligned}
$$

where $\mathrm{a}_{0}=\left[\begin{array}{c}\alpha_{1} \\ \vdots \\ \alpha_{\mathrm{m}}\end{array}\right], \mathrm{a}_{\mathrm{k}}=\frac{\partial \mathrm{a}_{0}}{\partial \mathrm{q}_{\mathrm{k}}}, \mathrm{c}_{\mathrm{ij}}=\left[\begin{array}{c}\mathrm{c}_{1}^{(\mathrm{ij})} \\ \vdots \\ \mathrm{c}_{\mathrm{m}}^{(\mathrm{ij})}\end{array}\right]$.

For instance, for a three-link manipulator $n=3$ and basic functions number $m=7$ :

$$
\alpha_{1}=1, \alpha_{2}\left(q_{2}\right)=\cos \mathrm{q}_{2}, \alpha_{3}\left(q_{2}\right)=\sin \mathrm{q}_{2}, \alpha_{4}\left(q_{3}\right)=\cos \mathrm{q}_{3},
$$
$\alpha_{5}\left(q_{3}\right)=\sin \mathrm{q}_{3}, \alpha_{6}\left(q_{2}, q_{3}\right)=\cos \left(\mathrm{q}_{2}+\mathrm{q}_{3}\right), \alpha_{7}\left(q_{2}, q_{3}\right)=$ $\sin \left(\mathrm{q}_{2}+\mathrm{q}_{3}\right)$. In this case, vector functions, included into (11) take the following form:

$$
a_{0}=\left[\begin{array}{c}
1 \\
\cos \left(q_{2}\right) \\
\sin \left(q_{2}\right) \\
\cos \left(q_{3}\right) \\
\sin \left(q_{3}\right) \\
\cos \left(q_{2}+q_{3}\right) \\
\sin \left(q_{2}+q_{3}\right)
\end{array}\right], a_{1}=\left[\begin{array}{l}
0 \\
0 \\
0 \\
0 \\
0 \\
0 \\
0
\end{array}\right], a_{2}=\left[\begin{array}{c}
0 \\
-\sin \left(q_{2}\right) \\
\cos \left(q_{2}\right) \\
0 \\
0 \\
-\sin \left(q_{2}+q_{3}\right) \\
\cos \left(q_{2}+q_{3}\right)
\end{array}\right],
$$

$$
a_{3}=\left[\begin{array}{c}
0 \\
0 \\
0 \\
-\sin \left(q_{3}\right) \\
\cos \left(q_{3}\right) \\
-\sin \left(q_{2}+q_{3}\right) \\
\cos \left(q_{2}+q_{3}\right)
\end{array}\right]
$$

With regard for (11) differential equations (10) are simplified to:

$$
\begin{aligned}
& \sum_{\mathrm{j}=1}^{\mathrm{n}}\left(\mathrm{a}_{0}^{\mathrm{T}} \mathrm{c}_{\mathrm{ij}}\right) \ddot{\mathrm{q}}_{\mathrm{j}}=-\left(\sum_{\mathrm{k}=1}^{\mathrm{n}} \mathrm{a}_{\mathrm{k}} \dot{\mathrm{q}}_{\mathrm{k}}\right)^{\mathrm{T}} \sum_{\mathrm{j}=1}^{\mathrm{n}} \mathrm{c}_{\mathrm{ij}} \dot{\mathrm{q}}_{\mathrm{j}},(i=1,2, \ldots, n) . \\
& +\frac{1}{2} \mathrm{a}_{\mathrm{i}}^{\mathrm{T}} \sum_{\mathrm{j}=1}^{3} \sum_{\mathrm{k}=1}^{3} \mathrm{c}_{\mathrm{jk}} \dot{\mathrm{q}}_{\mathrm{j}} \dot{\mathrm{q}}_{\mathrm{k}}+\mathrm{M}_{\mathrm{i}}
\end{aligned}
$$

System of $n$ ordinary differential equations (12) of $2 n$ order with initial conditions

$$
\mathrm{q}_{\mathrm{i}}(0)=\mathrm{q}_{\mathrm{i} 0}, \dot{\mathrm{q}}_{\mathrm{i}}(0)=\dot{\mathrm{q}}_{\mathrm{i} 0},(i=1,2, \ldots, n)
$$

represents symbolic model, describing dynamic behavior of a planar $n$-link manipulator at given instant $M_{i}(t, \mathrm{q}, \dot{\mathrm{q}})$ in the joints.

\section{Numerical Model Implementation}

For integration of differential equations system for manipulator dynamics (12) the decisive Runge-Kutta finitedifference scheme of 4-th order with constant time step was applied. The size of step was chosen during numerical studies. Differential equations of manipulator's dynamics are not considered as solved in reference to second derivatives, thus values of generalized accelerations $\ddot{q}_{1}, \ddot{q}_{2}$, $\ddot{q}_{3}$ on each time step resulted from solving linear system.

$$
\mathrm{A}(\mathrm{q}) \ddot{\mathrm{q}}=\mathrm{B}(t, \mathrm{q}, \dot{\mathrm{q}})
$$

where $\mathrm{A}(\mathrm{q})=\left\|a_{i j}(\mathrm{q})\right\|$ - inertia matrix of mechanism, B vector of the right hand side of the equation (12).

Calculation example cited below has following values of geometrical and inertial parameters of mechanism links: $m_{1}$ $=10 \mathrm{~kg}, m_{2}=10 \mathrm{~kg}, m_{3}=8 \mathrm{~kg} ; L_{k}=1 \mathrm{~m} ; I_{k}=5 m_{k} L_{k}{ }^{2} / 81$, $r_{k x}=L_{k} / 3, r_{k y}=-L_{k} / 9,(k=1,2,3)$.

Moment $M_{1}$, functioning in joint $O_{1}$, was given in a form

$$
M_{1}(t)=\left\{\begin{array}{c}
10 \mathrm{H} \cdot \mathrm{M}, \text { if } 0 \leq t<2 \mathrm{c} \\
-10 \mathrm{H} \cdot \mathrm{M}, \text { if } 2 \leq t<4 \mathrm{c} \\
0, \text { if } t \geq 4 \mathrm{c}
\end{array}\right.
$$

i.e. it corresponded to controlling moment, which provides the turn of a solid object about fixed axis through the terminal angle. 
For the moments functioning in joints $\mathrm{O}_{2}$ и $\mathrm{O}_{3}$, were accepted consequently:

$$
\begin{aligned}
& M_{2}=-c\left(q_{2}-q_{20}\right)-\mu \dot{q}_{2} \\
& M_{3}=-c\left(q_{3}-q_{30}\right)-\mu \dot{q}_{3}
\end{aligned}
$$

where $c=10 \mathrm{H} \cdot \mathrm{m} / \mathrm{rad}, \mu=20 \mathrm{H} \cdot \mathrm{m} / \mathrm{rad} \cdot \mathrm{c}^{-1}$.

First terms in the expressions (15) correspond to moments of joints' flexible response, obstructing reciprocal rotation of links, fixed in positions $q_{2}=q_{20}, q_{3}=q_{30}$. Addends in (14) match up moments of resistance forces in proportion to angular velocities of reciprocal link rotation.

Initial values of generalized coordinates and mechanism velocities:

$$
q_{i}(0)=q_{i 0}=0, \dot{q}_{i}(0)=\dot{q}_{i 0}=0,(\mathrm{i}=1,2,3) .
$$

Figure 2 represents the results of numeric equations integration (11) with initial conditions (16) and generalized forces (14), (15).

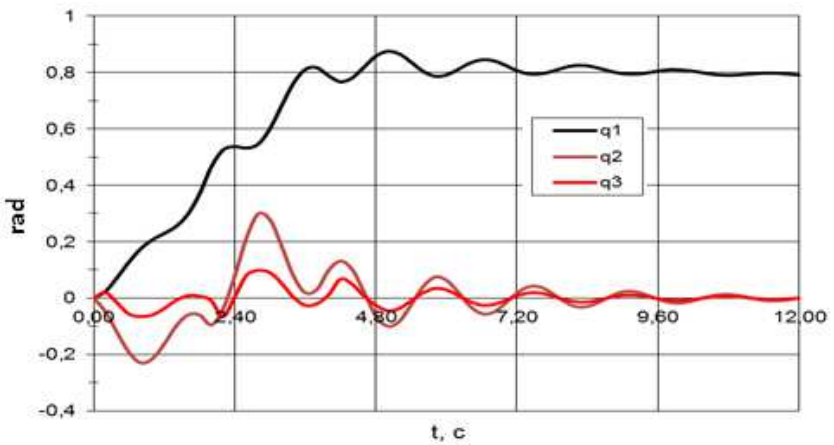

Figure 2. Generalized coordinates variation for three link mechanism: $q_{1}(t), q_{2}(t), q_{3}(t)$

Curves in figure 2 show generalized coordinates changes that take place in course of time $q_{1}, q_{2}, q_{3}$ consequently. Mechanism behavior corresponds to a desired rotation through the terminal angle. Noted oscillatory damping results from dissipative moments (14), functioning in joints $\mathrm{O}_{2}$ and $\mathrm{O}_{3}$.

The computer with Intel processor, which possesses operating frequency $2,8 \mathrm{GHz}$, at time step integration $\Delta t=$
$0,02 \mathrm{~s}$ is able to compute mechanism motion lasting $12 \mathrm{~s}$ in Mathcad 7.0 during about $1 \mathrm{~s}$ of machine time.

\section{Conclusion}

The way, recommended to form differential equations for dynamics of flat jointed multilink mechanism allows avoiding lengthy symbolic expressions [2] in order to compute elements of inertia matrix, as well as centrifugal and gyroscopic components. At the same time, during steps of numeric integration of dynamic equations, the calculation of current values of all components and equation coefficients at every time step is completed with explicit end formulas without employing recursive algorithms [5]. This method for describing flat joint mechanism with number of links $N>3$ seems to be of great value.

The paper is written in the context of assignment №2014/217 to carry out state projects in scientific field within basic part of state project for Ministry of Education and Science of the Russian Federation.

\section{References}

[1] Belousov I. R. Calculation of the robot manipulator dynamic equations. IAM RAS preprints №45, 2002.- 28p.

[2] Bosyakov S.M. Kinematic and dynamic modeling of mechanical systems. - Minsk: BSU, 2011.- 260 p.

[3] Zhuravlev E.A. Application of basic functions for description of manipulator dynamics. // Research. Technologies. Innovations.: collection of articles edited by Ivanov V.A. — Yoshkar-Ola: Mari STU, 2011.- P.107-110.

[4] Course of theoretical mechanics/ edited by Kolesnickov K.S - M.: Published by Bauman Moscow STU, 2005.- 736p.

[5] Walker M. W., Orin D. E., Efficient Dynamic Computer Simulation of Robotic Mechanisms, Trans. ASME, J. Dynamic Systems, Measurement \&Control, vol. 104, 1982, pp. 205-211. 